\title{
Erratum: Iatrogenic aortic insufficiency following concomitant septal myectomy and aortic valve replacement
}

\author{
Saiju Seo ${ }^{1}$, Jeesuk Sim ${ }^{1}$, Young Song ${ }^{1,2}$, Young Lan Kwak ${ }^{1,2,3}$, and Jong Wha Lee ${ }^{1,3}$ \\ ${ }^{1}$ Department of Anesthesiology and Pain Medicine, ${ }^{2}$ Anesthesia and Pain Research Institute, ${ }^{3}$ Division of Anesthesiology and Pain \\ Medicine, Cardiovascular Hospital, Yonsei University College of Medicine, Seoul, Korea
}

Korean J Anesthesiol 2013; 65(2): 172-173

The article by Seo S, et al. entitled, "Iatrogenic aortic insufficiency following concomitant septal myectomy and aortic valve replacement" (Korean J Anesthesiol 2013; 65(2): 172-173) contained an error in author's name.

\section{Before correction:}

Saija Seo, Jeesuk Sim, Young Song, Young Lan Kwak, and Jong Wha Lee

The correct information is found below:

Saiju Seo, Jeesuk Sim, Young Song, Young Lan Kwak, and Jong Wha Lee

The first author's name was misspelled as Saija Seo. The correct spelling is Saiju Seo.

The authors apologize for any inconvenience this mistake may have caused.

(ㄷ) This is an open-access article distributed under the terms of the Creative Commons Attribution Non-Commercial License (http:// creativecommons.org/licenses/by-nc/3.0/), which permits unrestricted non-commercial use, distribution, and reproduction in any medium, provided the original work is properly cited. 